\title{
ON SOME ASPECTS OF XUV SPECTROHELIOGRAMS*
}

\author{
R. Tousey, G. D. Sandlin, and J.D. Purcell \\ (E.O. Hulburt Center for Space Research, U.S. Naval Research Laboratory, \\ Washington, D.C., U.S.A.)
}

\begin{abstract}
A BSTR ACT
A comparison between XUV and Ca-K spectroheliograms for 9 dates from 1963 to 1967 showed an excellent correlation between plage intensities in Ca-K and HeII $304 \AA$, except for plages near the limb and a few others. Around the limb all but the highest ionization XUV emission lines form a bright ring, usually weaker over the poles. This is an unresolved combination of the limb-brightened emission from the quiet corona and high chromosphere, and emission extending into the corona above plages located as much as several days from limb passage.

In Fexv and XVI only the localized coronal emissions are observed; these vary in form and intensity from line to line. The 171-500 $\AA$ and white-light coronas, recorded on November 12, 1966, correlate well at low altitudes, but beyond $3^{\prime}$ the XUV corona becomes diffuse and without structure.
\end{abstract}

A comparison has been made between the extreme ultraviolet (XUV) solar images obtained since 1963 by the Naval Research Laboratory (NRL), and the corresponding $\mathrm{Ca}-\mathrm{K}$ spectroheliograms photographed by the McMath-Hulbert Observatory, giving special attention to the centres of activity and the corona. One rocket-borne instrument is a spectroheliograph, that produces a spectrum of monochromatic solar images, covering $\lambda 171-650 \AA$. Although there is much overlapping, the images in certain important lines are sufficiently separated to permit distinguishing between centres of activity. These lines are He II $304 \AA$, OIV $554 \AA$, O v $630 \AA$, Ne VII $465 \AA$, MgIX $_{3} 36 \AA$, Fexv $284 \AA$, and Fexvi $335,361 \AA$; arranged in this order they come from higher and higher levels in the solar atmosphere; therefore, viewed in these lines, the change in form and intensity of a centre of activity can be followed through the chromosphere into the corona. The resonance lines of FeIX-XIV between $171 \AA$ and $274 \AA$ are also present, with great intensity; but they lie so close together that the solar images are not well separated.

A second type of instrument, called an XUV heliograph, was first flown on July 27, 1966. This uses a paraboloidal mirror and three aluminum filters in series to form a broadband XUV solar image, including all emissions from $171 \AA$ to about $500 \AA$. The image has great intensity; with 30-sec exposures XUV coronal emission extending to $3 R_{\odot}$ has been recorded (Purcell et al., 1967).

Table 1 lists the dates on which spectroheliograms and heliograms have been ob-

* Presented by R. Tousey.

Kiepenheuer (ed.). Structure and Development of Solar Active Regions, 411-419. I.A.U. 
R. TOUSEY ET AL.

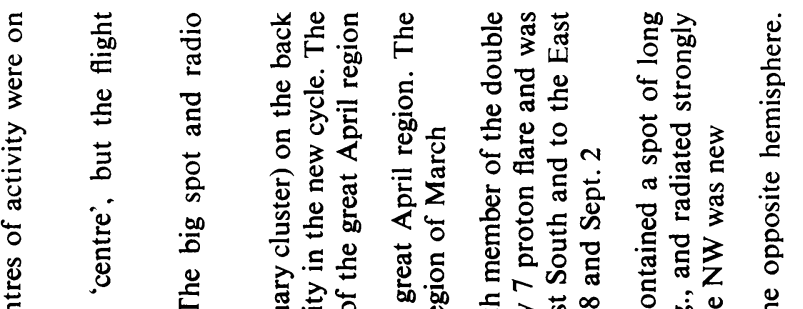

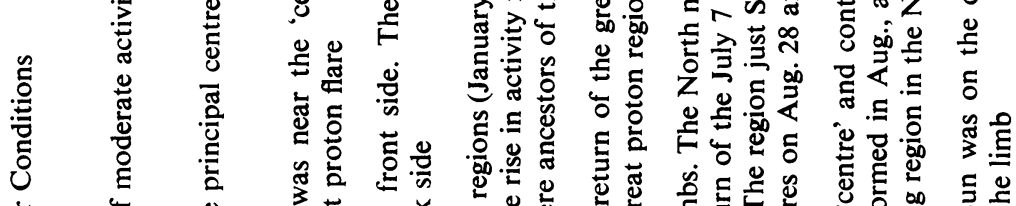

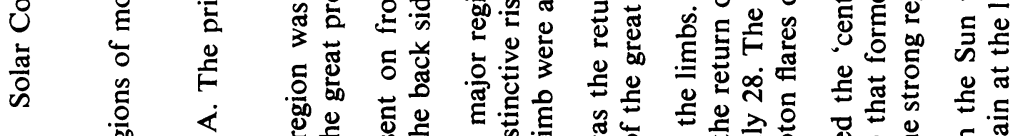

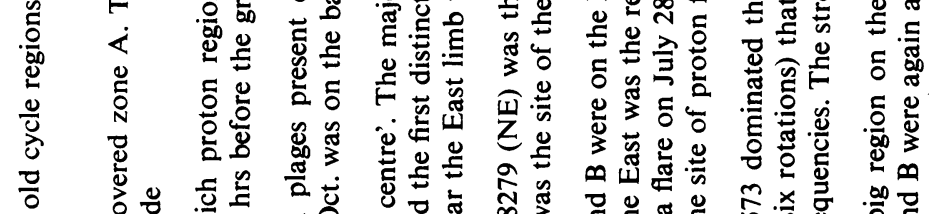

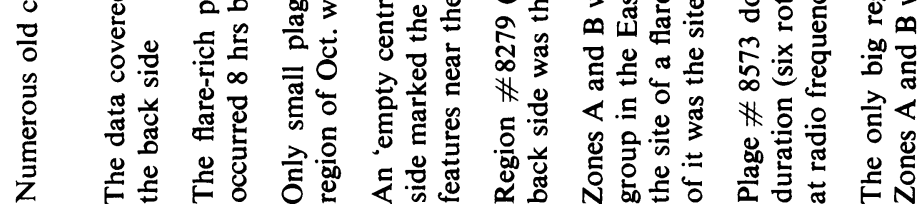

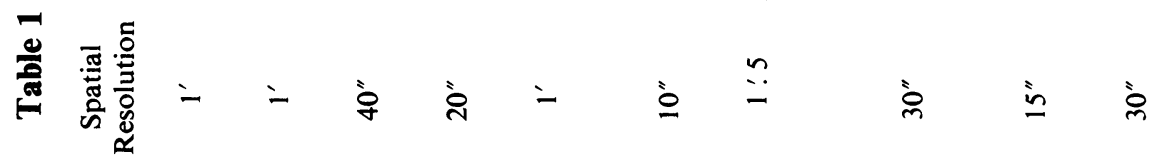

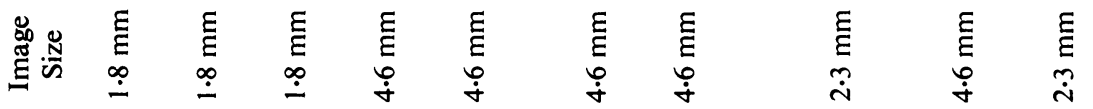

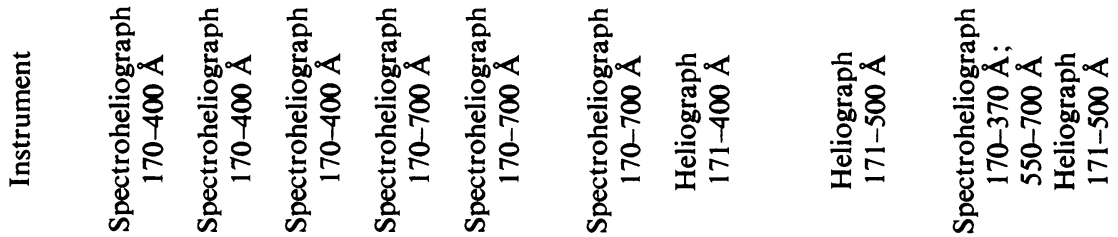

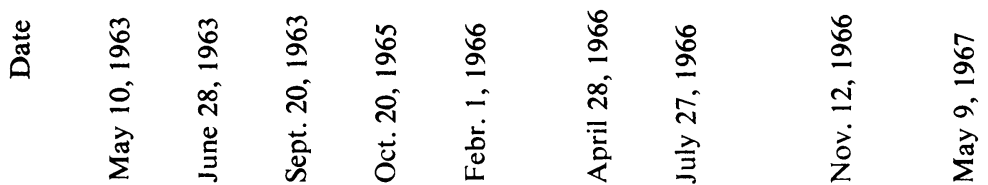


tained by NRL, and describes briefly the solar activity and the nature of the photographic results. For the purpose of making intensity correlations between the XUV and $\mathrm{Ca}-\mathrm{K}$ plages, an arbitrary intensity scale was set up, similar to the one which is used for Ca-K plages at the McMath-Hulbert Observatory. Plages were selected no farther than approximately $70^{\circ}$ from the central longitude. The photographic density of each active region in HeII $304 \AA$ was estimated relative to the average disk background on a scale rated: $1=$ just detectable, to $5=$ extremely bright. This was also done for the XUV heliograms.

When the HeII $304 \AA$ intensity values were plotted against the McMath-Hulbert values for the same regions in Ca-K, a linear relationship was observed with a scatter of \pm half a scale unit, excluding the exceptional cases to be discussed. The intensity estimates of the plages, as recorded by the broad-band XUV heliograph, also correlated well with Ca-K. The plages in He II $304 \AA$ are more intense, relative to the quiet background than in Ca-K, as was found for H-Lyman- $\alpha$ (Purcell and Tousey, 1961). For the higher ionization lines such as MgIX $368 \AA$, Fexv $284 \AA$, and Fexvi 335, $361 \AA$ there was no background against which to make a comparison; however, with a few exceptions, the plage intensities in the different lines increased or decreased together.

There were a few cases of plages not close to the limb that were clearly different in the XUV than in Ca-K. The most notable example is shown in Figure 1, where two of the best HeII $304 \AA$ images obtained as yet, with $10^{\prime \prime}$ spatial resolution, are compared with images in Ca-K and $\mathrm{H} \alpha$. The activity on April 28, 1966 lay in a belt in the North. The plage at $9{ }^{\circ} \mathrm{E}, 28^{\circ} \mathrm{N}$ is strong in Ca-K but is just detectable in He II; in $\mathrm{H} \alpha$ this feature is small and weak, and is surrounded by a ray-like absorption structure. This plage received no McMath-Hulbert number, but was the Western extension of plage \# 8278. Its position was about halfway between the two longitude zones of maximum activity.

Great differences between the XUV and the Ca-K images are observed for regions near and on the limb. For example, in Figure 2 two exposures with the XUV heliograph on July 27, 1966 are compared with Ca-K and $\mathrm{H} \alpha$. Over most of the disk the same plages are present, with similar shapes and intensities. Within 1 or 2 min of the limb and beyond the limb, however, the images show much more emission in the XUV than in Ca-K. The intense XUV emission in the NW is the most striking example; it is not present at all in Ca-K and $\mathrm{H} \alpha$. This activity is the coronal extension above a plage complex that was 2-3 days over the limb.

The bright, partial ring present in Figure 2 is a characteristic of all XUV heliograms and is much the same in images produced by X-rays of $\lambda<100 \AA$. It is composed of two parts, which cannot be fully separated without greater spatial resolution. One is just the corona above the quiet regions; the emitting layer is optically thin and is much more intense when viewed edge-on. The second contribution is from the corona above the active regions on or within a few days of limb passage. The ring is especially 


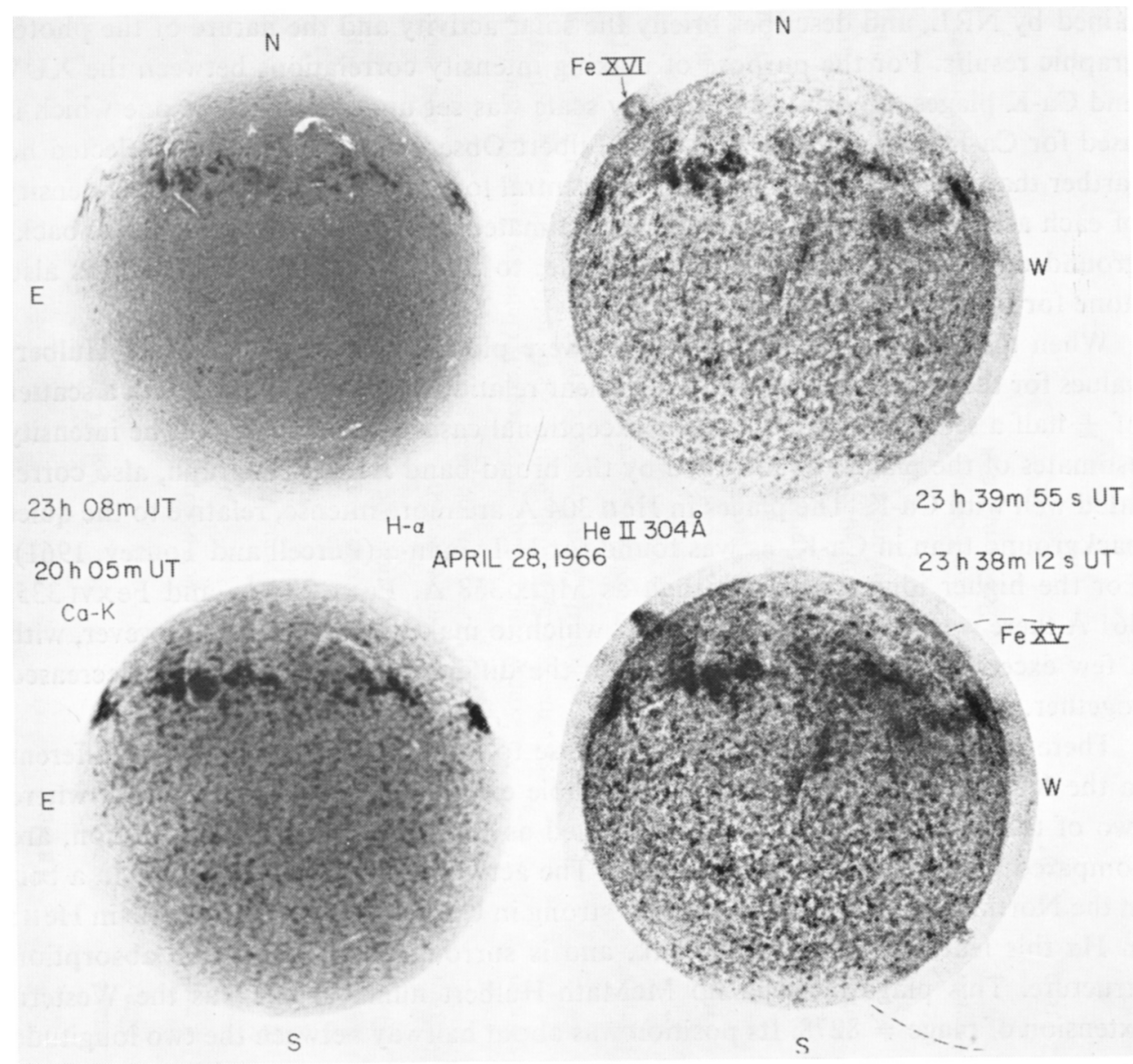

FIG. 1. Two HeII 304 A spectroheliograms obtained on April 28, 1966, compared with Ca-K from Mt. Wilson and Ha from Sacramento Peak. The Fexv 284 A image, whose position is shown, produces the plages that appear at the centre of the HeII image, and others that are in the West; several prominences are present in HeII, but FeXvi 335 A accounts for the large limb feature in the NE.

pronounced in all of the XUV images obtained by NRL because, for every flight date except September 20,1963, both zones A and B of maximum solar activity (DodsonPrince and Hedeman, 1967) lay close to the limb, and the disk as a whole showed a relatively 'empty centre'. Therefore, enhancements in the emission ring were prominent; for each enhancement an active centre that appeared to account for it was found either on the limb or within 1-5 days of limb passage.

The high-spatial resolution spectroheliograms of April 28, 1966 show several interesting limb features. In Figure 3 the range $240-340 \AA$ is reproduced, and in Figure 4 four particular images are brought together for comparison. 


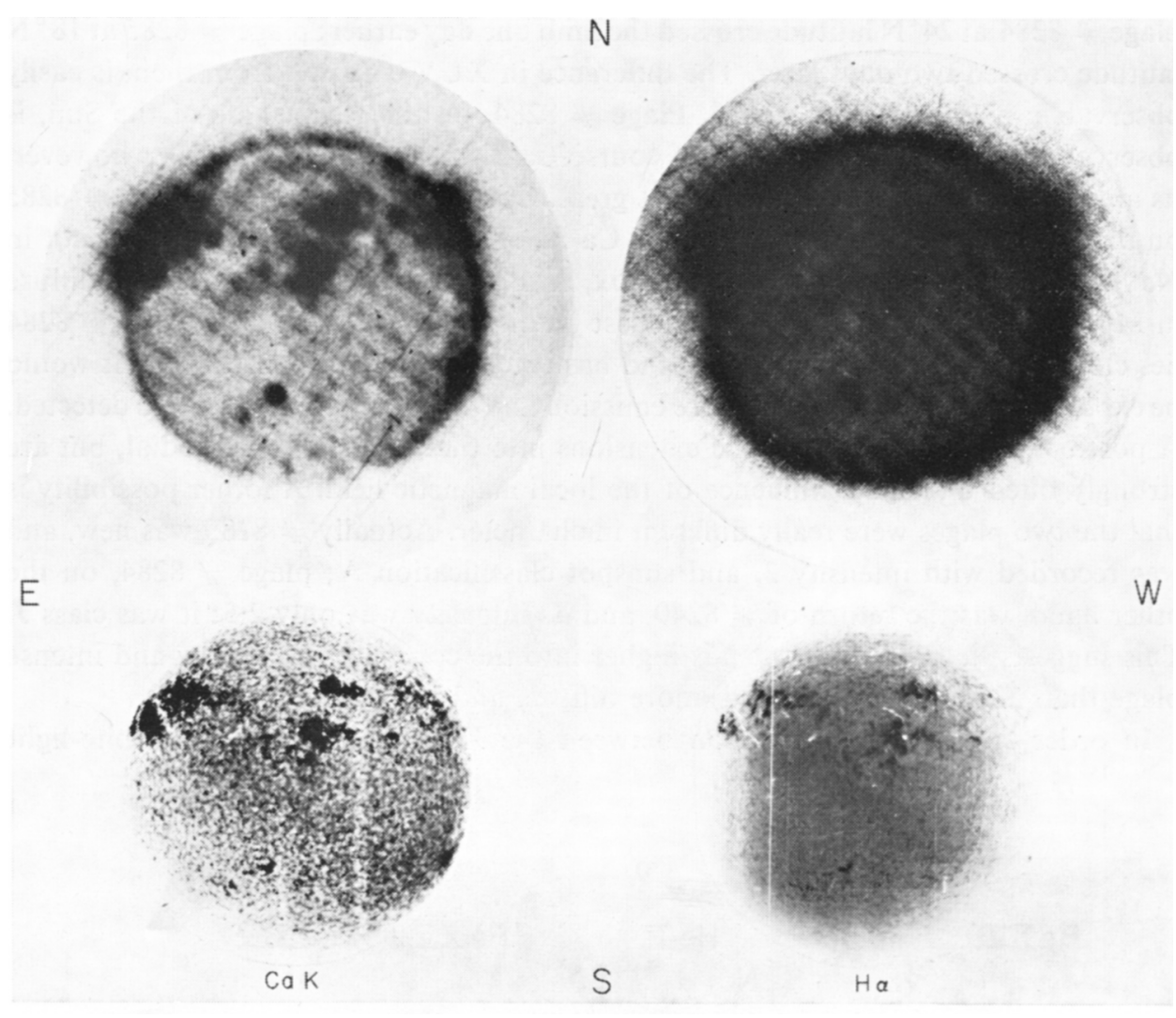

Fig. 2. Medium and long exposures with the XUV heliograph (171-500 A) on July 27, 1966, compared with $\mathrm{Ca}-\mathrm{K}$ and $\mathrm{H} \alpha$ from the Mt. Wilson Observatory. The grid pattern was caused by the mesh on which one of the aluminum filters was supported.

In Fexv $284 \AA$ and Fexvi $335 \AA$ there is coronal emission in the NW that extends to about $65^{\circ}$ apparent latitude and reaches $5^{\prime}$ beyond the limb. This emission is present also in HeII $304 \AA$, but in this case there is a possibility that it may be caused by Si XI $304 \AA$ instead of HeII (Smith, 1967). This coronal emission is believed to originate from a large active complex between latitudes $15^{\circ}$ and $35^{\circ}$, and lying $30^{\circ}-60^{\circ}$, heliocentric, over the limb; this was the great particle-emitting region of March 1966. The complex was assigned plage numbers 8262 and 8275 and had total area 7100 and intensity 3.5 during the preceding half-period; it was given numbers $8294,8299,8300$ during the succeeding half-period. Therefore the XUV emission region, seen in projection above the NW limb, was at a height above the photosphere of at least $3.5 \times 10^{5}$ $\mathrm{km}\left(0.5 R_{\odot}\right)$ and may have been observed to $10^{6} \mathrm{~km}\left(1.5 R_{\odot}\right)$.

Close to East-limb passage on April 28, 1966 there were two centres of activity; 
plage \# 8284 at $24^{\circ} \mathrm{N}$ latitude crossed the limb one day earlier; plage \# 8285 at $18^{\circ} \mathrm{N}$ latitude crossed two days later. The difference in XUV emission from them is easily observable in Figures 1, 3, and 4. Plage \# 8284, on the visible side of the Sun, is observable in all lines, including, of course $\mathrm{Ca}-\mathrm{K}, \mathrm{H} \alpha$, and $\mathrm{HeII}$. In Ov, however, its area is very small and its intensity is great. Coronal emission above plage \# 8285 on the back side cannot be seen in $\mathrm{H} \alpha, \mathrm{Ca}-\mathrm{K}, \mathrm{HeII}, \mathrm{OIV}, \mathrm{Ov}$, and probably not in Ne viI. It is conspicuous, however, in MgIX, Si XII, Fexv, and FexVI. It is most diffuse in MgIX, and appears to have the steepest gradient in FexVI. Because plage \# 8284 lies closer to the limb than \# 8285, and has twice the area (3900 vs. 1900), it would be expected to show as much or more emission above the limb, but none was detected. A possible explanation is that the extensions into the corona are not radial, but are strongly tilted under the influence of the local magnetic field. Another possibility is that the two plages were really different in character. Actually \# 8285 was new, and was recorded with intensity 3 , and sunspot classification A; plage \# 8284, on the other hand, was the return of \# 8240 , and its intensity was only $2 \cdot 5$; it was class $\mathrm{J}$. This suggests that emission extends higher into the corona above a new and intense plage than above one that is old, more diffuse, and less intense.

In order to make a comparison between the XUV images and the white-light

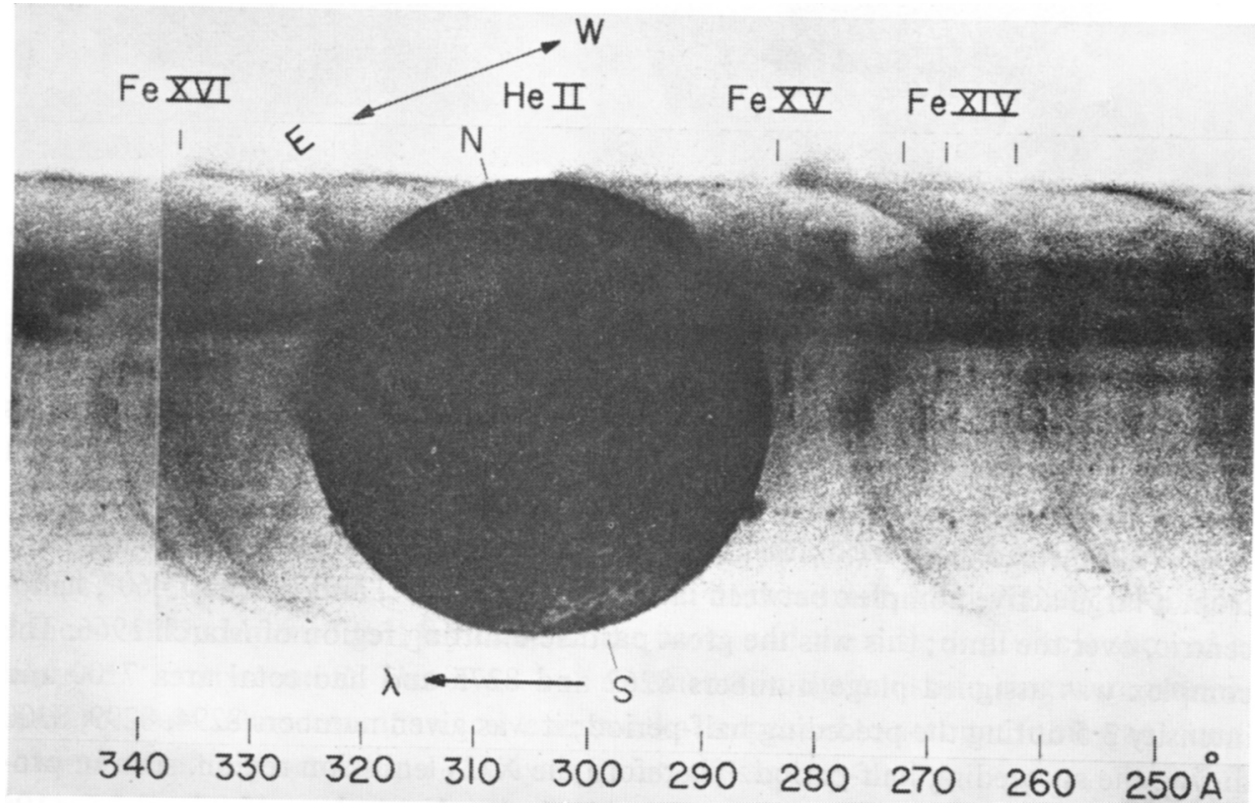

Fig. 3. The section, 250-340 $A$, of the spectroheliogram of longest exposure on April 28, 1966 , printed so as to reproduce the coronal emission in the $N W$, associated with FeXVI $335 \AA$, FeXV $284 A$, $\mathrm{He}$ II and SiXI $304 A$, and faintly with FeXIV. 


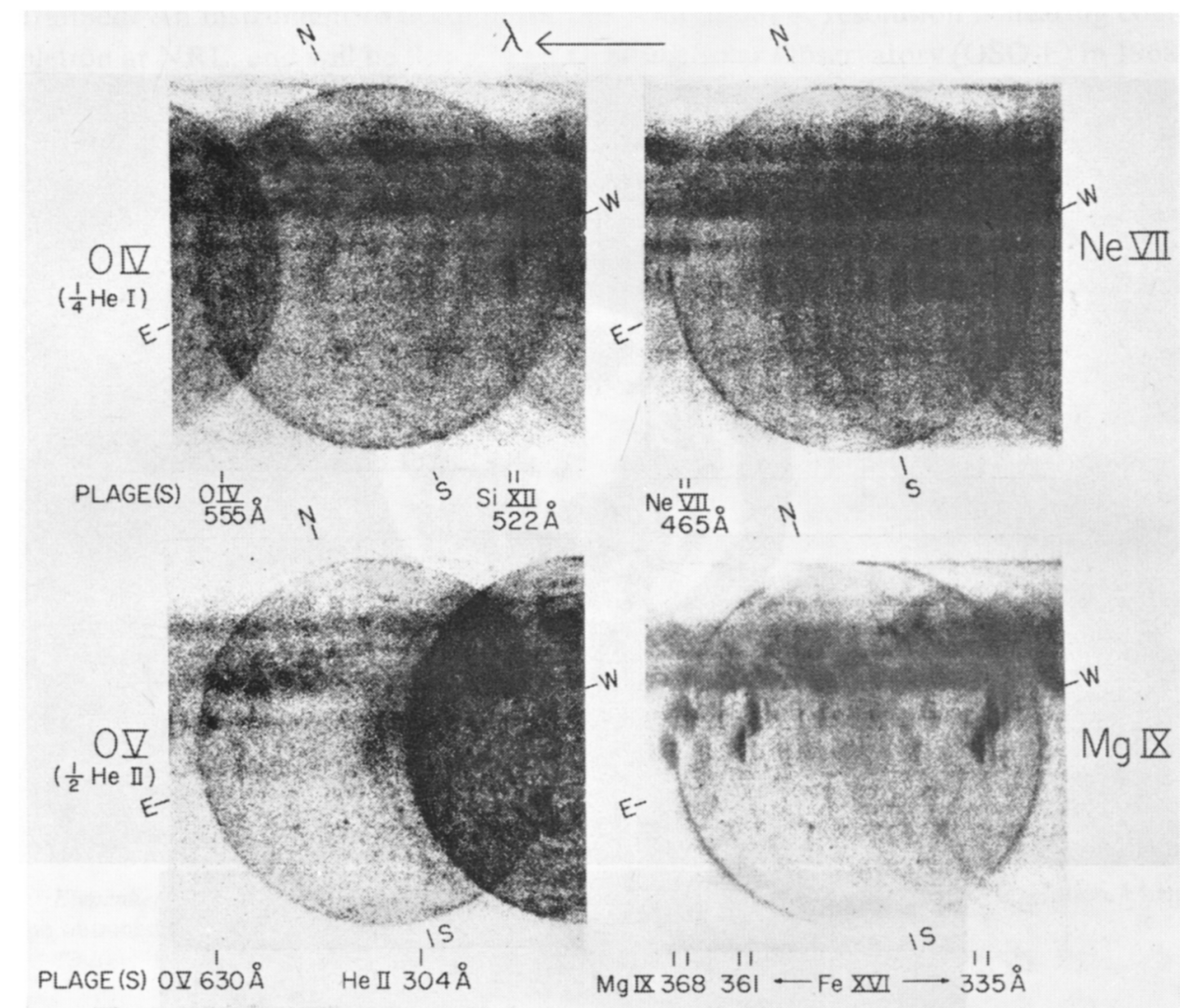

FIG. 4. Sections of the April 28, 1966 spectroheliogram covering $O$ IV $554 A, O$ v $630 A$, Ne VII $465 A$, and MgIX 368 A. Also present are a small part of the HeI $584 A$ image, and half of the second-order image of HeII 304 A. The East-limb features discussed in the test are indicated.

corona, a flight was scheduled for November 12, 1966, the day of the total eclipse in South America. Figure 5 shows Newkirk's (1967) beautiful eclipse photograph, but with the centre removed and an XUV image introduced to scale. Below, is another XUV image that was exposed for a longer time. Close to the limb the XUV emission matches very well the detail in the white-light corona. This is to be expected, because collisional excitation of the XUV resonance lines follows $n_{\mathrm{e}}^{2}$, and optical excitation follows $n_{\mathrm{e}}$. Farther out, however, the two types of corona are less similar; the long XUV exposure shows no structure or streamers, but only a diffuse emission, correlating in an approximate fashion with the white-light corona.

The XUV spectroheliograms and heliograms contain a great deal of information about the structure of the chromosphere and corona. But to make use of them a day to day series is required. The spatial resolution should be the greatest that can be 

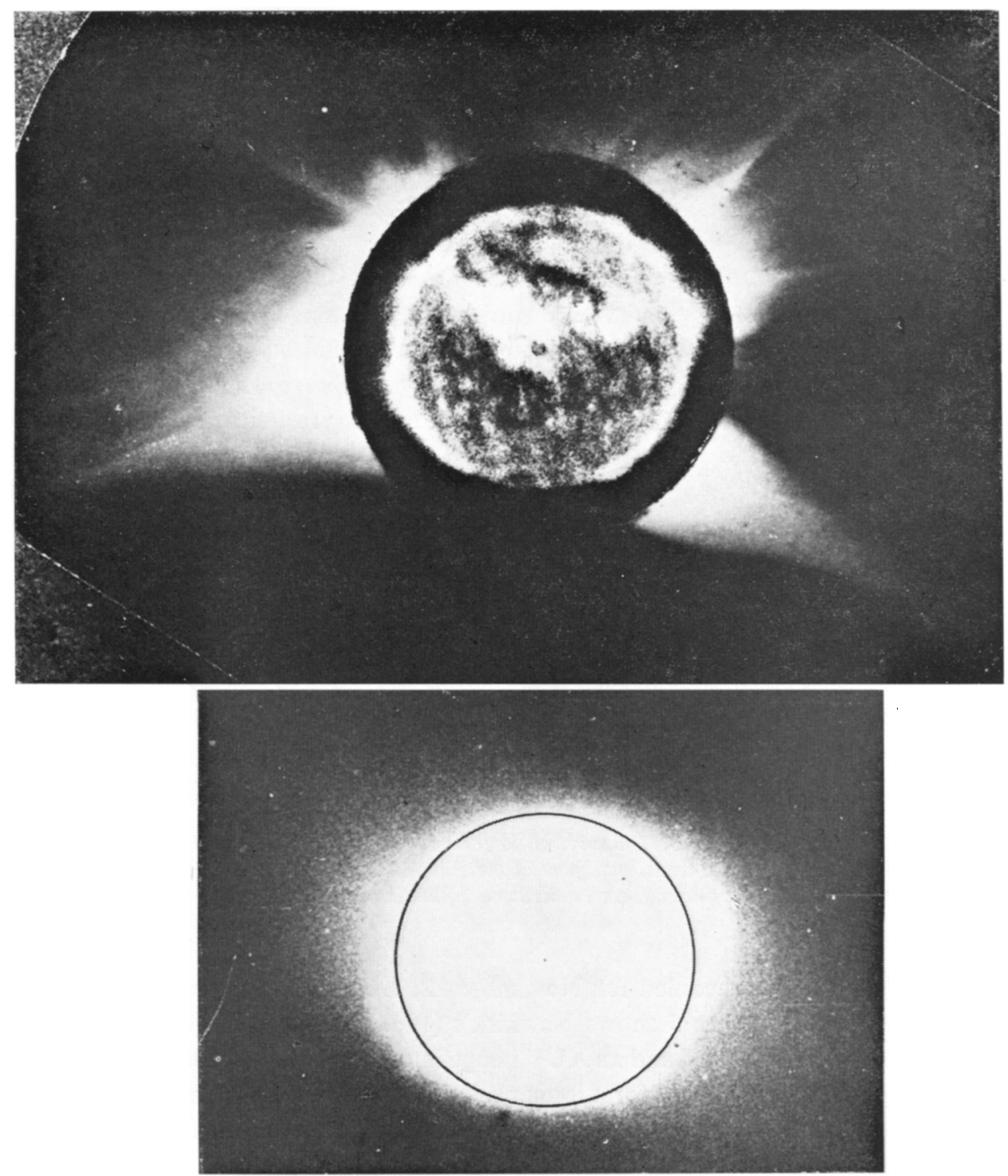

FIG. 5. The white-light corona, photographed during the November 12, 1966 eclipse by the Bolivian Expedition of the High Altitude Observatory and The Johns Hopkins University, with the centre replaced by an XUV heliogram obtained on the same date by the Naval Research Laboratory. The second heliogram, of long exposure, shows the extension of XUV emission into the corona. North is at the top and East to the left. 
attained. An instrument to accomplish this with about $1^{\prime}$ resolution is nearing completion at NRL, and will be flown in an Orbiting Solar Observatory (OSO-F) in 1968. This will be followed, 1 to 2 years later, by a photographic spectroheliograph with very high spatial resolution, that will be included in the Astronomical Telescope Mount of the Apollo Applications Program, and will operate over one to two solar rotations.

\section{Acknowledgments}

We wish to express our sincere appreciation to Dr. Helen Dodson-Prince and to Miss Ruth Hedeman of the McMath-Hulbert Observatory for making available their solar data and much helpful advice.

\section{References}

Dodson-Prince, H., Hedeman, E. R. (1967) Annals of the IQSY.

Newkirk, G.Jr. (1967) Sky and Telescope, 33, 136.

Purcell, J.D., Tousey, R. (1961) Mém. Soc. R. Sc. Liège, 4, 274.

Purcell, J.D., Tousey, R., Koomen, M.J. (1967) in Space Research, VIII.

Smith, T.S. (1967) discussion at the meeting of the Amer. Astron. Soc., Boulder, Colo., Oct. 3, 1966.

\section{DISCUSSION}

Kiepenheuer: I would like to congratulate Dr. Tousey on the beautiful angular resolution which he obtained in the EUV from a rocket.

Tousey: This was just luck! 